\title{
Doped Overoxidized Polypyrrole Microelectrodes as Sensors for the Detection of Dopamine Released from Cell Populations
}

Sasso, Luigi; Heiskanen, Arto; Diazzi, Francesco; Dimaki, Maria; Castillo, Jaime; Vergani, Marco; Landini, Ettore; Raiteri, Roberto; Ferrari, Giorgio ; Carminati, Marco

Total number of authors:

13

Published in:

Analyst

Link to article, DOI:

10.1039/C3AN00085K

Publication date:

2013

Document Version

Early version, also known as pre-print

Link back to DTU Orbit

Citation (APA):

Sasso, L., Heiskanen, A., Diazzi, F., Dimaki, M., Castillo, J., Vergani, M., Landini, E., Raiteri, R., Ferrari, G., Carminati, M., Sampietro, M., Svendsen, W. E., \& Emnéus, J. (2013). Doped Overoxidized Polypyrrole Microelectrodes as Sensors for the Detection of Dopamine Released from Cell Populations. Analyst, 138, 36513659. https://doi.org/10.1039/C3AN00085K

\section{General rights}

Copyright and moral rights for the publications made accessible in the public portal are retained by the authors and/or other copyright owners and it is a condition of accessing publications that users recognise and abide by the legal requirements associated with these rights.

- Users may download and print one copy of any publication from the public portal for the purpose of private study or research.

- You may not further distribute the material or use it for any profit-making activity or commercial gain

- You may freely distribute the URL identifying the publication in the public portal 


\section{Accepted Manuscript}

\section{Analyst}

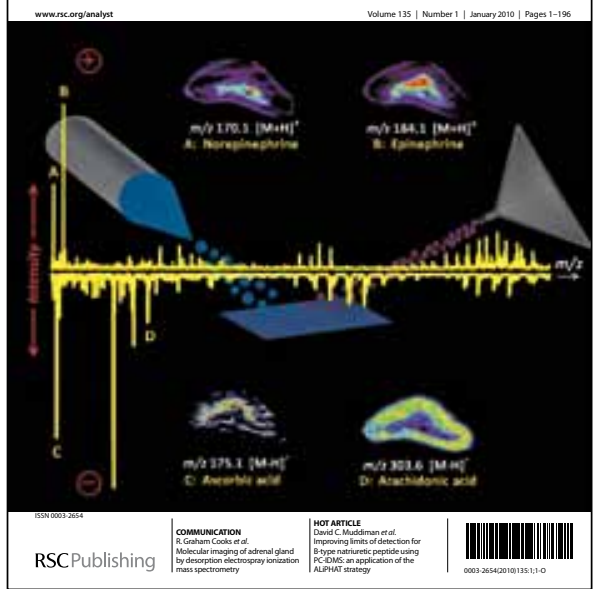

This is an Accepted Manuscript, which has been through the RSC Publishing peer review process and has been accepted for publication.

Accepted Manuscripts are published online shortly after acceptance, which is prior to technical editing, formatting and proof reading. This free service from RSC Publishing allows authors to make their results available to the community, in citable form, before publication of the edited article. This Accepted Manuscript will be replaced by the edited and formatted Advance Article as soon as this is available.

To cite this manuscript please use its permanent Digital Object Identifier $\left(\mathrm{DOl}^{\oplus}\right)^{\text {, }}$ which is identical for all formats of publication.

More information about Accepted Manuscripts can be found in the

\section{Information for Authors.}

Please note that technical editing may introduce minor changes to the text and/or graphics contained in the manuscript submitted by the author(s) which may alter content, and that the standard Terms \& Conditions and the ethical guidelines that apply to the journal are still applicable. In no event shall the RSC be held responsible for any errors or omissions in these Accepted Manuscript manuscripts or any consequences arising from the use of any information contained in them. 


\title{
Doped Overoxidized Polypyrrole Microelectrodes as Sensors for the Detection of Dopamine Released from Cell Populations
}

\author{
Luigi Sasso, ${ }^{a, \S}$ Arto Heiskanen, ${ }^{a}$ Francesco Diazzi, ${ }^{a, \#}$ Maria Dimaki, ${ }^{a}$ Jaime Castillo-León, ${ }^{a}$ Marco \\ Vergani, ${ }^{b}$ Ettore Landini, ${ }^{c}$ Roberto Raiteri, ${ }^{c}$ Giorgio Ferrari, ${ }^{b}$ Marco Carminati, ${ }^{b}$ Marco Sampietro, ${ }^{b}$ \\ ${ }_{5}$ Winnie E. Svendsen ${ }^{a}$ and Jenny Emnéus ${ }^{a}{ }^{a}$ \\ Received (in XXX, XXX) Xth $X X X X X X X X X 20 X X$, Accepted Xth XXXXXXXXX 20XX \\ DOI: 10.1039/b000000x
}

A surface modification of interdigitated gold microelectrodes (IDEs) with a doped polypyrrole (PPy) film for detection of dopamine released from populations of differentiated PC12 cells is presented. A thin PPy layer was potentiostatically electropolymerized from an 10 aqueous pyrrole solution onto electrode surfaces. The conducting polymer film was doped during electropolymerization by introducing counter ions in the monomer solution. Several counter ions were tested and the resulting electrode modifications were characterized electrochemically to find the optimal dopant that increases sensitivity in dopamine detection. Overoxidation of the PPy films was shown to contribute to a significant enhancement in sensitivity to dopamine. The changes caused by overoxidation in the electrochemical behavior and electrode morphology were investigated using cyclic voltammetry and SEM as well as AFM, respectively. The optimal 15 dopant for dopamine detection was found to be polystyrenesulfonate anion ( $\mathrm{PSS}^{-}$). Rat pheochromocytoma (PC12) cells, a suitable model to study exocytotic dopamine release, were differentiated on IDEs functionalized with an overoxidized PSS-doped PPy film. The modified electrodes were used to amperometrically detect dopamine released by populations of cells upon triggering cellular exocytosis with an elevated $\mathrm{K}+$ concentration. A comparison between the generated current on bare gold electrodes and gold electrodes modified with overoxidized doped PPy illustrates the clear advantage of the modification, yielding 2.6 -fold signal amplification. The results also 20 illustrate how to use cell population based dopamine exocytosis measurements to obtain biologically significant information that can be relevant in, for instance, the study of neural stem cell differentiation into dopaminergic neurons.

\section{Introduction}

${ }_{25}$ Conducting polymers have gathered attention from the global scientific community since the discovery of the conductive properties of doped polyacetylene in the late $70^{\prime}$ 's. ${ }^{1}$ Their use in analytical sensors has been moved forward mainly because of the flexibility they provide, offering both intrinsic properties and the 30 ability for functionalization, rendering the polymer matrix sensitive to, e.g., $\mathrm{pH}$, inorganic ions, organic molecules and gases. ${ }^{2}$ Particularly polypyrrole (PPy) has been attractive because of the properties of the pyrrole monomer, having good water solubility, as well as being readily oxidizable and commercially 35 available. PPy has been widely used in electrochemical sensors because it facilitates biomolecule immobilization onto electrode surfaces, ${ }^{3,4}$ can be functionalized, ${ }^{5}$ is biocompatible, ${ }^{6}$ and provides protection against electrochemical fouling. ${ }^{7,8}$

The complex matrix structure of an electropolymerized film 40 facilitates the doping of PPy with specific ions and organic molecules, ranging from small ions to large organic molecules such as enzymes, by entrapping the dopants in the film during its deposition onto an electrode surface. Several studies have been presented in literature illustrating the effects of doping on the

45 properties of a PPy film, which are dependent on the dopant and the electropolymerization conditions. ${ }^{9-12}$

The catecholamine, dopamine, is produced in vivo in different parts of the peripheral and central nervous system (CNS) as well as in the medulla of adrenal glands. This molecule is involved in 50 a wide range of physiological processes, including hormonal regulation, cardiovascular and renal functions, ${ }^{13}$ sleep, feelings of reward, memory and learning ${ }^{14}$ as well as control of motor functions. $^{15}$ In the CNS, dopamine functions as a neurotransmitter, being released by presynaptic neurons upon 55 arrival of an action potential and received by the G-protein coupled receptors of the post synaptic neurons, initiating a cascade of biochemical processes. Different neurological disorders, e.g., schizophrenia and Parkinson's disease (PD), can be caused by abnormality in the dopaminergic activity in the ${ }_{60} \mathrm{CNS}$. Schizophrenia is related to increased activation of dopamine receptors. ${ }^{16}$ Although gradual loss of dopamine is a typical trait of old age, its complete depletion due to death of dopaminergic neurons in the CNS region substantia nigra causes impairment of motor functions, which is a characteristic symptom 65 of $\mathrm{PD}$, the second most common neurodegenerative disease related to old age. ${ }^{17}$

Because of the tight correlation between dopamine and brain functions, detection of dopamine release from cells in vitro and in vivo has been a fundamental topic in brain research. ${ }^{18}$ Dopamine 70 and other catecholamines can be detected electrochemically upon oxidation on an electrode surface, ${ }^{19}$ resulting in numerous studies that have revealed, for instance, the fundamental mechanism of neurotransmitter release in the process known as exocytosis. 
Inside neurons, neurotransmitters are sequestered in vesicles that upon stimulation are fused with the plasma membrane in a $\mathrm{Ca}^{2+}$ influx mediated process, resulting in the release of the vesicular contents into the extracellular environment. Electrochemical 5 investigations have demonstrated that neurotransmitters are released as small packages upon fusion of one vesicle at a time, ${ }^{20}$ known as quantal release. Typically, micromanipulated carbon fiber microelectrodes (CFMEs) have been applied in electrochemical detection of released dopamine from single ${ }_{10}$ cells. ${ }^{20}$ However, during recent years, metal electrode systems based on lithographic microsensor technology have gained increasing attention. ${ }^{21}$ Aside from suitability for single cell detection, microfabricated metal electrodes have the advantage of facilitating monitoring of dopamine release from populations of 15 adherently growing cells. This approach gives possibility for fast statistically significant screening of cell populations with respect to their dopaminergic properties. It can effectively answer biologically relevant questions in the field of neural stem cell research where the ability of chosen differentiation conditions 20 need to be evaluated in terms of an increase in the number of dopaminergic neurons in a cell population. ${ }^{22,23}$

Dopamine detection at a bare metal electrode surface can be hampered by electrode fouling due to polymerization of dopamine on the electrode surface ${ }^{24}$ during electrooxidation and 25 by the presence of negatively charged interferents, such as ascorbate. ${ }^{25}$ To overcome the influence of these factors, different electrode modifications, e.g., thiol self-assembled monolayers $(\mathrm{SAMs})^{26}$ and overoxidized $\mathrm{PPy},{ }^{25}$ have been utilized. In this paper, we describe PPy modification of interdigitated gold 30 microelectrodes based on an optimized counter-ion doping during electropolymerization and overoxidation of the polymer layer to increase sensitivity to dopamine and minimize electrode fouling during detection of dopamine released by populations of differentiated PC12 cells.

\section{${ }_{35}$ Results and discussion}

All the electrodes used in this work were fabricated onto a silicon-based microchip in gold using standard cleanroom technology. Each set of interdigitated electrodes used as working electrode (WE) was coupled with its own counter (CE) and 40 reference electrode (RE) present on the microchip, as shown in Fig. 1A. This individual addressability of the WEs allowed selective formation of PPy on each WE using a specific counter ion.

\section{Polypyrrole electropolymerization and influence of 45 overoxidation}

In potentiostatic polymerization, the formation of PPy is initiated by the creation of pyrrole radical-cation intermediates and further on dimmers and oligomers that are adsorbed at the electrode surface, initiating the formation of polymer chains in different 50 nucleation points. ${ }^{27}$ Allowing this process to proceed for a certain time, PPy films with varying morphological and electrochemical properties are created on the electrode depending on the time poised at the applied potential and the used counter ion.

Subjecting PPy films to several potential cycles in the presence 55 of oxygen, reaching sufficiently high overpotentials (up to $1 \mathrm{~V}$ ), results in irreversible overoxidation of the polymer film, blocking the electron transfer in the nitrogen-containing aromatic rings of the backbone and forming oxygen-containing functionalities such as carbonyl $^{28}$ and carboxylic groups ${ }^{29}$. Although these 60 functionalities reduce the conductivity of the PPy film, the resulting electronegativity attracts cations to the surface of the film, therefore rendering the electrode more sensitive to, for instance, dopamine, as well as more permselective, eliminating anionic interferents, e.g. ascorbate and dopamine metabolite ${ }_{65}$ dihydroxyphenylacetic acid. ${ }^{30}$

When evaluating the influence of counter ion doping on the morphology of electropolymerized PPy, two cases have to be considered: one where the dopant counter ion is a relatively small anion, as in the case of $\mathrm{Cl}^{-}, \mathrm{SO}_{4}{ }^{2-}$ and $\mathrm{PO}_{4}{ }^{3-}$, and one where the 70 dopant molecule is large enough to have an effect on the final polymer film morphology and thickness, as for the large anions PSS $^{-}$and DS. The nominal thickness of the obtained PPy films can be estimated based on Faraday's law of electrolysis by integrating the amperometric current-time traces for the 75 polymerization experiments, assuming $100 \%$ current efficiency, an average polymer density of $1.5 \mathrm{~g} / \mathrm{cm}^{3}$ and the involvement of 2 electrons in the polymerization reaction. ${ }^{31}$ The calculated average thickness of the obtained PPy film in the presence of small counter-ions was $44 \mathrm{~nm} \pm 1 \mathrm{~nm}$ (s.e.m.; $\mathrm{n}=24$ ), while in the case 80 of large counter-ions the estimated thickness was $82 \mathrm{~nm} \pm 5 \mathrm{~nm}$ (s.e.m.; $n=12$ ). A larger variation in thickness was found for PPy films polymerized in the presence of large dopant counter-ions.

The effect of overoxidation on the morphology of the polymer film created by electropolymerization can be seen in the SEM and ${ }_{85}$ AFM images shown in Fig. 2. In the case of both small and large counter ion doping, overoxidation of PPy results in flattening of the film (compare Fig. 2A,B with 2C,D and Fig. 2E,F with $2 \mathrm{G}, \mathrm{H})$. The AFM images show that the flattening resulting from the overoxidation also reduces the roughness of the film, 90 therefore affecting its porosity. Hence, even if the overoxidation yields a loss of conductivity in the film, the thinning of this film reduces the electron transfer distance between the electrochemically active analyte and the underlying gold electrode, which retains its conductive metallic properties.

95 Fig. 2 clearly illustrates the differences between the morphologies when using small and large counter ions. Small dopant ions make the resulting PPy film tightly packed onto the surface of the electrode (Fig. 2A,B), whereas large dopant ions render the PPy film relatively rough with a more amorphous 100 surface (Fig. 2E,F). This visual investigation of the PPy surfaces can be related to differences in the calculated thickness of the PPy films doped with small and large counter ions.

A characteristic increase in sensitivity to dopamine due to overoxidation of PPy can be seen in Fig. 3, which shows cyclic 105 voltammograms of a PPy-modified gold IDE set before and after overoxidation. Before overoxidation, the neutral state PPy modification results in voltammetric peaks corresponding to dopamine oxidation and reduction hidden under currents generated by redox processes of the polymer and non-faradaic 110 processes caused by an increased double layer capacitance, making the anodic current rise with an almost ohmic behavior. After overoxidation of the PPy film, dopamine electron transfer occurs directly to the underlying gold IDEs. The effect of the double layer capacitance is drastically decreased and the faradaic 115 processes of the neutral state PPy are eliminated, which facilitates 
observation of clear peaks corresponding to oxidation (top: anodic peak) and reduction (bottom: cathodic peak) of dopamine.

\section{Counter ion doping}

Depending on the counter-ion used as dopant and particularly on ${ }_{5}$ its size, the electropolymerized PPy film will exhibit different morphologies and electrochemical behavior. ${ }^{32-34}$ The presence of negative ions in the solution helps to stabilize the pyrrole radicalcation intermediate by interacting with the pyrrole rings during electropolymerization and maintaining local electroneutrality in 10 the solvent environment. ${ }^{35}$ Incorporating an anion in the polymer matrix therefore changes the obtained overall structure of the film; this effect increases in the case of large anions, such as DS and polymeric PSS .

The electrochemical response to dopamine of electrodes with 15 doped overoxidised PPy films was investigated. Fig. 4A shows cyclic voltammograms of a $100 \mu \mathrm{M}$ dopamine solution on gold IDEs modified with overoxidized PPy doped with the different counter ions, categorized as large (DS-, $\left.\mathrm{PSS}^{-}\right)$or small $\left(\mathrm{Cl}^{-}, \mathrm{SO}_{4}{ }^{2-}\right.$, $\left.\mathrm{PO}_{4}{ }^{3-}\right)$. Evaluation of the obtained peak currents immediately 20 leads to the conclusion that an electrode modified with a PPy film electropolymerized using large counter ions as dopants, such as $\mathrm{DS}^{-}$or $\mathrm{PSS}^{-}$, yields a significantly higher response than those having small counter ions, such as $\mathrm{Cl}^{-}, \mathrm{SO}_{4}{ }^{2-}$ and $\mathrm{PO}_{4}{ }^{3-}$ (for a full comparison of all the counter ions used, see $\mathrm{ESI}+$ ). In fact, an 25 overoxidized PPy film doped with small counter ions has a response similar to that of a non-doped overoxidized PPy film, indicating that the small counter ions do not enhance the signal response and, therefore, neither the sensitivity of the electrodes. However, an overoxidized PPy film clearly enhances the 30 response in comparison with the ones recorded using bare gold IDEs. This lack of difference between signals from a non-doped and a small counter ion doped PPy film can be explained by the overoxidation process, which, as mentioned above, introduces electronegative functionalities in the film. These can repel small 35 negative counter ions entrapped in the polymer matrix, whereas large counter ions remain entrapped in the polymer film, ${ }^{32}$ further enhancing the attraction to positively charged analytes, such as dopamine.

The dopant counter ion that contributed to the highest 40 electrode response and hence to the greatest sensitivity to dopamine in all experiments was PSS $^{-}$; clear peaks could be observed in cyclic voltammograms down to dopamine concentrations in the $\mathrm{nM}$ range (see Fig. 4B). The observed increase in response to dopamine is related to the formation of 45 carbonyl- and carboxylic acid functionalities. ${ }^{30}$ The effect of these functionalities can be seen in an analogous manner as has been shown in the case of thiol SAMs as a modification to improve dopamine electrochemistry (thiol SAMs having a partially deprotonated carboxylic tail group, e.g., thioctic acid ${ }^{36}$ 50 and mercaptopropionic acid $\left.{ }^{26}\right)$. It is believed that the presence of carbonyl- and carboxylic acid functionalities orient the electroactive hydroxylic groups of dopamine molecules toward the electrode surface, in this way enhancing the oxidation of dopamine.

55 In the case of overoxidized PPy, an additional effect can be proposed to further explain the observed current amplification during oxidation of dopamine. Despite the fact that the overoxized PPy film becomes morphologically flattened in comparison with the initial conducting PPy film, it is a 360 dimensional polymer network comprising carbonyl- and carboxylic acid functionalities as well as retained counter ions (PSS ${ }^{-}$with dispersed negative charges. This structure can more effectively accommodate the diffusing dopamine in the vicinity of the electrode surface, increasing the fraction of oxidized 65 dopamine.

Aside from simply orienting the hydroxylic groups of dopamine molecules toward the electrode surface or accommodating more dopamine molecules in the vicinity of the electrode surface, the 3-dimensional structure with dispersed 70 negative charges can also decrease the freedom of movement of the tail of dopamine molecules with the protonated positively charged amino group. This can be seen as a combined steric and electrostatic hindrance, limiting the possibility of intramolecular cyclization, which is the prerequisite for polymerization of 75 dopamine on an electrode surface. ${ }^{24}$ Hence, overoxidized PPy can serve as a protection against electrode fouling that otherwise could decrease current response. However, since steric hindrance in the packing of the PPy matrix may leave gaps between the individual PPy chains on the electrode surface, there is, 80 nevertheless, a possibility for electrode fouling, which can be observed during continued potential cycling (results not shown).

\section{Exocytosis measurements}

Since the optimization experiments showed that a PSS $^{-}$doped overoxidized PPy film is, among all the options tested in this 85 work, the best electrode modification in terms of sensitivity, this specific modification was chosen for the detection of cellular dopamine release.

PC12 cells were chosen because of their wide use as models to study neuronal functions, such as exocytosis. ${ }^{37}$ This cell line was 90 created several decades ago from a rat adrenal pheochromocytoma with the aim of creating a cell model for neurochemical and neurobiological studies. ${ }^{38}$ These cells are known for their reliable release of dopamine, and have been used for implantation into neuronal tissue. ${ }^{39,40}$

95100,000 cells $/ \mathrm{cm}^{2}$ was chosen as the cell density for seeding PC12 cells onto the electrode chip (seen in Fig. 1B) in order to facilitate differentiation for several days without reaching too high a confluency. This precaution is significant in order to allow proper formation of the characteristic axon-like outgrowths. As 100 shown in Fig. 5A, the PPy electrode modification did not hinder proper cell differentiation. Since PC12 cells require an adhesion factor on a growth substrate, the PPy-modified electrodes were coated using physisorbed laminin, which has been shown to facilitate good differentiation of different cell types, including 105 neurons and PC12 cells. ${ }^{41}$ A PC12 cell population differentiating on one set of IDEs can be seen in Fig. 5B. The PC12 cells extended the axon-like outgrowths on the IDEs, seemingly preferring the modified electrode surface to the rest of the chip (see Fig. 5C). Cells on the laminin modified electrodes showed 110 the typical cell adhesion, differentiation morphology and confluency expected for $\mathrm{PC} 12$ cells. $^{42}$ This electrode configuration is optimal for accommodating the axon-like outgrowths when monitoring release of neurotransmitters from a whole population of differentiating cells. The function of 115 population-based exocytosis monitoring from growing nondifferentiated PC12 cells using $30-\mu \mathrm{m}$ disk electrodes has been 
demonstrated previously. ${ }^{43}$ However, such an electrode structure and dimension is limited to primarily address the cell bodies and not the axon-like outgrowths. Hence, disk electrodes are mainly suitable for recordings from growing PC12 cells, which have 5 round cell bodies. Even when the diameter of disk electrodes is increased to $100 \mu \mathrm{m},{ }^{44}$ a population of differentiating PC12 cells cannot be accommodated well on them due to the elongated morphology of the cells' outgrowths.

Fig. 6 depicts amperometric current-time traces generated on 10 IDEs during a measurement, as well as the baseline for a control measurement where no cells were present on the IDE. As expected, the amplitude of the recorded current generated by dopamine oxidation on an IDE modified with overoxidized PSS-doped PPy (PPy/PSS) was significantly higher than that obtained 15 on a bare gold IDE. In detection of single vesicle exocytotic events, various parameters can be extracted from recorded current-time traces (spikes), such as the integrated area of the current spikes (the charge Q yielding the amount of molecules released during each event), the maximum oxidation current, 20 half-width $t_{1 / 2}$, and the rise-time. ${ }^{45}$ In measurements of dopamine release from a cell population, the recorded current-time traces are composed of a large number of superimposed single vesicle events. In this case, the most significant parameter is the total charge, which, in an analogous manner as in the case of single 25 vesicle measurements, is proportional to the detected amount. The average generated charge on bare gold IDEs having an average cell population of $217 \pm 5$ cells (s.e.m.; $n=10$ ) (based on visual counting under microscope) was $8.7 \pm 0.6 \mathrm{nC}$ (s.e.m.; $\mathrm{n}=5$ ), while on PPy/PSS modified IDEs, having cell populations 30 of the same dimension as on bare gold IDEs, the average generated charge was $23 \pm 2 \mathrm{nC}$ (s.e.m.; $\mathrm{n}=5$ ).

The different quantities of charge generated on bare gold IDEs and PPy/PSS modified IDEs upon dopamine detection do not, however, mean that the cells residing on bare gold electrodes and ${ }_{35} \mathrm{PPy} / \mathrm{PSS}$ modified electrodes released different amounts of dopamine. Instead, the PPy/PSS modified IDEs show an increased sensitivity to dopamine, indicating 2.6-fold amplification. This is in accordance with the value previously published for PPy modified CFMEs. ${ }^{30}$ It is significant to point out 40 that measurements conducted on bare electrodes and PPy/PSS modified electrodes are not directly comparable since, for cell populations of the same dimension, different levels of charge are being recorded. On the other hand, the increase in detection sensitivity makes the electrode modification highly suitable for 45 monitoring dopamine release under conditions, where very small changes need to be detected, such as in vivo monitoring of the dynamics of dopamine release in the rat brain ${ }^{30}$ and characterization of conditions leading to differentiation of neural stem cells to dopaminergic neurons. ${ }^{22,23}$

50 The results presented in this paper highlight the novelty of overoxidized PPy as an electrode modification for detection of cellular dopamine release. The modification is readily prepared, resulting in a reproducible electrode behavior (for details of the effect of the prerequisite electrode cleaning, see ESI $\dagger$ ), and 55 improves the capability to monitor small amounts of released molecules. Furthermore, the modification is stable under cell culture conditions for long-term experiments and shows no adversary effects on the differentiated cells.

Although PSS$^{-}$as the dopant clearly provided the highest
60 amplification in dopamine detection, the results obtained in this work when using $\mathrm{DS}^{-}$as the dopant also indicated amplification. This is in accordance with the previously published findings. ${ }^{25}$ However, the dimension of $\mathrm{DS}^{-}$ion is very critical. Due to its size, it has an increased possibility to leak out from the 65 overoxidized PPy film during long-term experiments, which can cause harmful effects on the cultured/differentiated cells. This was indicated as decreased and irreproducible cellular dopamine release (results not shown). Hence, for a reliable application of overoxidized PPy as an electrode modification for monitoring 70 cellular dopamine release, PSS$^{-}$is considered the optimal dopant among those tested in this work during PPy electropolymerization.

\section{Experimental}

\section{Chemicals}

75 Potassium chloride (BioXtra), sodium sulfate (ReagentPlus), monobasic potassium phosphate (cell culture tested), sodium dodecyl sulfate (DS', Mw $288.4 \mathrm{~g} / \mathrm{mol}$ (molecular biology grade), poly(sodium 4-styrenesulfonate) ( $\mathrm{PSS}^{-}$) (average $\mathrm{Mw} \sim 70,000$ $\mathrm{Da}, 30 \%$ wt. in water), hydrochloric acid (BioReagent), 80 magnesium chloride hexahydrate (BioReagent), calcium chloride dihydrate (BioReagent), sodium chloride (BioReagent), glucose (BioXtra), 4-(2-hydroxyethyl)piperazine-1-ethanesulfonic acid (HEPES) (1M solution; BioReagent), 2-(3,4dihydroxyphenyl)ethylamine hydrochloride (Dopamine), 3-(3,485 dihydroxyphenyl)-L-alanine (L-DOPA), sodium hydroxide (BioXtra), pyrrole (Reagent), hydrogen peroxide (30\% in water), potassium hydroxide (semiconductor grade), polyethylenimine (PEI) (branched, average $\mathrm{Mw} \sim 25,000$ ), nerve growth factor- $\beta$ from rat (NGF), laminin from Engelbreth-Holm Swarm murine 90 sarcoma basement membrane, horse serum (HS), glutaraldehyde $(25 \%$ in $\mathrm{H} 2 \mathrm{O}$, specially purified for use as an electron microscopy fixative), and water (cell culture tested) were purchased from Sigma-Aldrich Corporation (St. Louis, MO, USA). Dulbecco's Modified Eagle Medium (DMEM)/Ham's 95 Nutrient Mixture F12 with GlutaMAX (DMEM/F12), TrypsinEDTA $(0.05 \%)$ and penicillin/streptomycin $(\mathrm{P} / \mathrm{S})$ were purchased from Life Technologies Ltd (Paisley, UK). Foetal bovine serum (FBS) and cell culture tested phosphate buffered saline (PBS) were from Biowest S.A.S. (Nuaillé, France) and Lonza Group 100 Ltd (Basel, Switzerland), respectively. All solutions used for electropolymerization and electrochemical characterization were prepared in ultra pure water (resistivity $18.2 \mathrm{MOhm} \cdot \mathrm{cm}$ ) from a Milli-Q ${ }^{\circledR}$ water purification system (Millipore Corporation, Billerica, MA, USA).

\section{${ }_{105}$ Solutions, buffers and media}

Electropolymerization was done using $0.05 \mathrm{M}$ pyrrole (used as received) solution in water. Dopant counter-ions were added in a $10-\mathrm{mL}$ aliquot of the pyrrole solution to obtain concentration of $0.1 \mathrm{M}\left(\mathrm{Cl}^{-}, \mathrm{SO}_{4}{ }^{2-}, \mathrm{PO}_{4}{ }^{3-}\right.$, or $\left.\mathrm{DS}^{-}\right)$or $10 \mathrm{mg} / \mathrm{mL}\left(\mathrm{PSS}^{-}\right)$. Dopamine 110 solutions for sensor characterization were prepared by diluting a $1 \mathrm{mM}$ stock solution prepared in nitrogen purged PBS (purged for at least 20 minutes before use). Purging of the stock solution was continued throughout the sensor characterization. Each dilution (e.g. $100 \mu \mathrm{M}, 10 \mathrm{nM}$ ) was prepared immediately before use. 115 Buffers for exocytosis measurements were prepared using cell 
culture tested water. The low $\mathrm{K}^{+}$buffer contained $10 \mathrm{mM}$ HEPES, $5 \mathrm{mM}$ glucose, $1.2 \mathrm{mM} \mathrm{Mg}{ }^{2+}, 2 \mathrm{mM} \mathrm{Ca}^{2+}, 150 \mathrm{mM} \mathrm{Na}^{+}$, and $5 \mathrm{mM} \mathrm{K}^{+}$. In the high $\mathrm{K}^{+}$buffer, the concentration of $\mathrm{Na}^{+}$and $\mathrm{K}^{+}$were $5 \mathrm{mM}$ and $450 \mathrm{mM}$, respectively. Both buffers were 5 sterile filtered before use. All cell media were prepared using DMEM/F12. The growth medium was supplemented with $15 \%$ HS, $2.5 \% \mathrm{FBS}, 1 \% \mathrm{P} / \mathrm{S}$, and $0.5 \%$ HEPES. The differentiation medium had a lowered serum contents $(0.5 \% \mathrm{HS}$ and $0.5 \%$ FBS $)$. The L-DOPA $(100 \mu \mathrm{M})$ medium was prepared by adding L10 DOPA from a $10-\mathrm{mM}$ stock solution in PBS into the differentiation medium. The stock solution was prepared by first dissolving L-DOPA $(40 \mathrm{mg} / \mathrm{ml})$ in $0.5 \mathrm{M} \mathrm{HCl}$ followed by dilution with PBS and sterile filtration.

\section{Electrode microchip fabrication}

15 The microchips having 12 electrode sets, each comprising an interdigitated electrode (IDE), a counter electrode (CE) and a reference electrode (RE), all in gold (see Fig. 1), were fabricated using standard cleanroom-based micromachining techniques. A $670 \mathrm{~nm}$ layer of thermal oxide $\left(\mathrm{SiO}_{2}\right)$ was grown on a silicon 20 wafer (one side polished) in a drive-in furnace at $1050{ }^{\circ} \mathrm{C}$ in the presence of water vapor (wet growth). The gold structures, i.e. electrodes, leads and contact pads, were photolithographically defined in positive photoresist (AZ® ${ }^{\circledR}$ 5214E from MicroChemicals GmbH, Ulm, Germany) using an image reversal 25 process, followed by metal deposition $(10 \mathrm{~nm}$ thick $\mathrm{Ti}$ adhesion layer and $150 \mathrm{~nm}$ thick gold layer) through electron beam evaporation and lift-off in acetone. Prior to metal deposition, the $\mathrm{SiO}_{2}$ was etched for $100 \mathrm{~s}$ in the areas having opened resist using buffered HF solution to form ca. $150 \mathrm{~nm}$ deep isotropic 30 undercuts $^{46}$ that eliminate the formation of lift-off ears at the edges of the metal structures. The non-active gold areas (the leads connecting the electrodes to the contact pads) were passivated with $500 \mathrm{~nm}$ thick silicon nitride deposited using plasmaenhanced chemical vapor deposition. A second photolithography 35 step coupled with reactive ion etching of the silicon nitride was used to expose the active electrode areas and contact pads. Removal of the final photoresist was achieved by ultrasonication in acetone followed by intermediate rinsing with ethanol and final rinsing with deionized water.

${ }_{40}$ Polypyrrole electropolymerization, overoxidation and characterization

For the electropolymerization and electrode characterization, the electrode chips were placed in a micromilled poly(methyl methacrylate) (PMMA) holder (for details see ESI $\dagger$ ). The holder ${ }_{45}$ formed a $500-\mu \mathrm{L}$ vial on top of the electrode chip to facilitate liquid handling during experiments. Interconnections between the electrode chip and the potentiostat (1010A eight-channel potentiostat from $\mathrm{CH}$ Instruments, Austin, TX, USA) were obtained using a tailor-made PCB having gold plated spring 50 loaded pins (Mill-Max Mfg. Corp., Oyster Bay, NY, USA). Before use, the electrode chips were cleaned for 10 minutes in a solution containing $50 \mathrm{mM} \mathrm{KOH}$ and $25 \% \mathrm{H}_{2} \mathrm{O}_{2}$, followed by a potential sweep on the IDE working electrodes (WE) from -200 $\mathrm{mV}$ to $-1200 \mathrm{mV}$ in $50 \mathrm{mM} \mathrm{KOH}$ at $50 \mathrm{mV} / \mathrm{s}$ to remove the gold 55 oxides formed during the chemical cleaning. ${ }^{47}$ The polypyrrole (PPy) electrode modification was achieved by potentiostatic electropolymerization (10 seconds at $700 \mathrm{mV})$ with or without dopant counterions. The electropolymerization potential was chosen to obtain a neutral state PPy film before proceding with 60 the overoxidation, which occurs at around $900 \mathrm{mV}$ (data not shown). The IDEs of the microchip were used as WEs together with the $\mathrm{RE}$ and $\mathrm{CE}$ from each respective electrode set. Overoxidation of the PPy layer was carried out by cyclic voltammetry in a $0.1 \mathrm{M} \mathrm{NaOH}$ solution by applying 50 cycles in ${ }_{65}$ a potential window from 0 to $1000 \mathrm{mV}$ at the sweep rate of 100 $\mathrm{mV} / \mathrm{s}$. The electrochemical behavior of the doped PPy-modified electrodes was characterized by cyclic voltammetry in a potential window from 0 to $700 \mathrm{mV}$ at a potential sweep rate of $50 \mathrm{mV} / \mathrm{s}$ in solutions having various dopamine concentrations. A 70 morphological comparison of the polymer layer was done by low-vacuum scanning electron microscopy (SEM) using a FEI Nova 600 NanoSEM system (FEI Company, Oregon, USA) at 5 $\mathrm{kV}$ (spot size 4.0). Atomic Force Microscopy (AFM) characterization was carried out with a Veeco di CP-II 75 microscope (Veeco Instruments Inc., Plainview, NY, USA). The results showing the average thickness of the obtained PPy films are presented $+/$ - standard error of mean (s.e.m.), with $n$ being the number of characterized IDEs.

\section{Cell culturing and differentiation}

${ }_{80}$ The cell-based experiments were conducted using rat pheochromocytoma (PC12) cells from Deutsche Sammlung von Microorganismen und Zellkulturen $\mathrm{GmbH}$ (Braunschweig, Germany). Initially, PC12 cells were grown for 48 hours in PEIcoated T25 culture flasks from Nunc A/S (Roskilde, Denmark) 85 using growth medium. PEI coating was done at room temperature for 2 hours using sterile filtered $50 \mu \mathrm{g} / \mathrm{mL}$ PEI diluted in PBS followed by rinsing twice with PBS. 24 hours prior to seeding the cells onto an electrode chip, the growth medium was changed for the differentiation medium to initiate the cellular response to 90 NGF. Sterilization of all microfabricated cell culture substrates and materials was done by immersing them in $0.5 \mathrm{M} \mathrm{NaOH}$ for 30 minutes followed by rinsing with PBS trice. Electrode chips were coated with laminin $(20 \mu \mathrm{g} / \mathrm{mL}$ laminin diluted in PBS $)$ in a Petri dish for 2 hours to ensure cellular adhesion. Adsorption of 95 laminin, and hence adhesion of cells, only in the central part of an electrode chip where the electrode sets are located was achieved using a hydrophobic pen from Dako Denmark A/S (Glostrup, Denmark). The pre-differentiated PC12 cells were rinsed with PBS and trypsinized for 5 minutes followed by 5 -minute 100 centrifugation at $850 \mathrm{rpm}$ at $20{ }^{\circ} \mathrm{C}$. The cell pellet was resuspended in the differentiation medium and the cells were seeded onto the coated electrode chips with a surface density of $10^{5}$ cells $/ \mathrm{cm}^{2}$. All cell culturing and differentiation was done in an incubator at $37^{\circ} \mathrm{C}$ in a humidified atmosphere of $5 \% \mathrm{CO}_{2} / 95 \%$ 105 air. SEM imaging of the differentiated cells was done using a FEI Nova 600 NanoSEM system after fixation for 1 hour with $2 \%$ glutaraldehyde solution diluted in PBS followed by rinsing with PBS (twice for $15 \mathrm{~min}$ ) and cell culture tested water (twice for 5 min). Cell counting on microchips was done using a Zeiss Axio 110 Imager M1m microscope equipped with an AxioCam MRc5 computer controlled CCD camera (Carl Zeiss AG, Göttingen, Germany).

\section{Exocytosis measurements}

After 4 days of differentiation, the differentiation medium was 
replaced by the L-DOPA medium and the cells were kept in the incubator for one hour to increase the dopamine load in the vesicles. $^{48}$ Before conducting the exocytosis measurements, each electrode chip with the differentiated PC12 5 cells was placed in a micromilled PMMA holder (for details see $\mathrm{ESI} \dagger$ ) to facilitate contact between the individual electrode sets and a tailor-made 24-channel potentiostat with a dedicated acquisition software. ${ }^{49}$ The open vial in the holder facilitated the addition of the necessary buffer solutions during exocytosis 10 measurements. The medium was immediately replaced by $160 \mu \mathrm{L}$ of the low $\mathrm{K}^{+}$buffer to record a baseline for the measurements. Each array of interdigitated WEs was poised at $400 \mathrm{mV}$ vs. the gold RE adjacent to the array. After a stable baseline had been recorded, exocytosis was triggered by pipetting $80 \mu \mathrm{L}$ of the high ${ }_{15} \mathrm{~K}^{+}$buffer directly into the vial to elevate the $\mathrm{K}^{+}$concentration to $150 \mathrm{mM} .^{50}$ The current-time traces corresponding to the oxidation of the dopamine released by the cells were obtained shortly after triggering the exocytosis. Recording of the exocytotic events was done simultaneously on each IDE array. Exocytosis experiments 20 were carried out on 3 different electrode chips, each having nonmodified IDE arrays and IDE arrays modified with overoxidized PSS $^{-}$-doped PPy. All the amperometric recordings were done at room temperatrure $\left(\sim 22{ }^{\circ} \mathrm{C}\right)$. All calculated results from cellbased measurements are presented $+/$ - (s.e.m.), with $n$ being the 25 number of IDEs having cell populations subjected to exocytosis measurements.

\section{Conclusions}

This work demonstrates the obtained enhanced sensitivity in dopamine detection by using polystyrene sulfonate (PSS ${ }^{-}$) doped 30 electropolymerized and overoxidized polypyrrole (PPy) film as an electrode modification. The presented investigation on how overoxidation and doping of the conducting PPy film with different counter-ions influences the resulting morphology and the sensitivity to dopamine led to the proposal of three possible 35 factors contributing to the enhancement of dopamine detection: a three-dimensional polymer network with oxygen functionalities i) can guide the orientation of the catechol moiety, facilitating an improved electrochemical behavior previously shown for thiol modifications, ii) increase the fraction of detected dopamine, 40 resulting in current amplification, and iii) decrease the possibility of electrode fouling caused by dopamine polymerization. The application of PPy/PSS electrode modification to detection of released dopamine from populations of differentiated rat pheochromocytoma (PC12) cells demonstrates how the optimized 45 and characterized electrode modification provides a basis for future biological studies on, for instance, characterization of neural stem cell differentiation to dopaminergic neurons.

\section{Acknowledgement}

This work was supported by the EU FP7 project EXCELL 50 (NMP4-SL-2008-214706). A.H. additionally acknowledges Ørsted postdoctoral grant and Lundbeck Foundation grant no. R69-A6408 for financial support.

\section{Notes and references}

\author{
${ }^{a}$ Department of Micro- and Nanotechnology, Technical University of \\ 55 Denmark, Ørsted Plads 345E, 2800 Kgs. Lyngby, Denmark. Fax: +45 \\ 4588 7762; Tel: +454525 5741; E- \\ mail:winnie.svendsen@nanotech.dtu.dk \\ ${ }^{b}$ Department of Electronics and Information Technologies, Politecnico di \\ Milano, Via Golgi 42, 20133 Milano, Italy \\ ${ }_{60}{ }^{c}$ Department of Biophysical and Electronic Engineering, University of \\ Genova, Via Opera Pia 11A, 16145 Genova, Italy \\ $\$$ Present address: MacDiarmid Institute for Advanced Materials and \\ Nanotechnology; Biomolecular Interaction Centre, School of Biological \\ Sciences, University of Canterbury, Private Bag 4800, Christchurch \\ 65 8140, New Zealand \\ \# Present address: STMicroelectronics, Via Tolomeo 1, 20010 Cornaredo, \\ Italy \\ $\dagger$ Electronic Supplementary Information (ESI) available: A cyclic \\ voltammetry characterization of the IDEs in ferri/ferrocyanide solution; \\ 70 an illustration of the complete analytical setup; cyclic voltammetry \\ response comparison for individual counter ions. See \\ DOI: $10.1039 / \mathrm{b} 000000 \mathrm{x} /$
}

1 C.K. Chiang, C.R. Fincher, Jr., Y.W. Park, A.J. Heeger, H. 75 Shirakawa, E.J. Louis, S.C. Gau and A.G. MacDiarmid, Phys. Rev. Lett., 1977, 39, 1098-1101.

2 U. Lange, N.V. Roznyatovskaya and V.M. Mirsky, Anal. Chim. Acta, 2008, 614, 1-26.

3 P.N. Bartlett and J.M. Cooper, J. Electroanal. Chem., 1993, 362, 112.

4 A. Ramanavicius, A. Ramanaviciene and A. Malinauskas, Electrochim. Acta, 2006, 51, 6025-6037.

5 C.M. Hangarter, M. Bangar and A. Mulchandani, N.V. Myung, J. Mater. Chem., 2010, 20, 3131-3140.

856 P.M. George, A.W. Lyckman, D.A. LaVan, A. Hedge, Y. Leung, R. Avasare, C. Testa, P.M. Alexander, R. Langer and M. Sur, Biomaterials, 2005, 26, 3511-3519.

7 A. Galal, N.F. Atta, J.F. Rubinson, H. Zimmer and H.B. Mark, Jr., Anal. Lett., 1993, 26, 1361-1381.

908 T.J. Sata, J. Chem. Soc. Chem. Comm., 1993, 14, 1122-1124.

9 J.L. Brédas, B. Thémans, J.G. Fripat, M.M. André and R.R. Chance, Phys. Rev. B., 1984, 29, 6761-6773.

10 M. Zagórska, H. Wycislik and J. Przyluski, Synth. Met., 1987, 20, 259-268.

9511 S. Sadki, P. Schottland, N. Brodie and G. Sabouraud, Chem. Soc. Rev., 2000, 29, 283-293.

12 M. Ates and A.S. Sarac, Prog. Org. Coat., 2009, 66, 337-358.

13 M. Velasco and A. Luchsinger, Amer. J. Therap., 1998, 5, 37-43.

14 J-M. Beaulieu and R.R. Gainetdinov, Pharmacol. Rev., 2001, 63, 182-217.

15 J.J. Jankovic, Neurol. Neurosurg. Psychiatry, 2008, 79, 368-376.

16 A. Abi-Dargham, J. Rodenhiser, D. Printz, Y. Zea-Ponce, R. Gil, L.S. Kegels, R. Weiss, T.B. Cooper, J.J. Mann, R.L. Van Heertum, J.M. Gorman and M. Laruelle, Proc. Natl. Acad. Sci. USA, 2000, 97, 8104-8109.

17 W. Dauer and S. Przedborski, Neuron, 2003, 39, 889-909.

18 R.M. Wightman, L.J. May and A.C. Michael, Anal. Chem., 1988, 60, 769A-779A.

19 R.N. Adams, Anal. Chem., 1976, 48, 1126A-1138A.

11020 R.M. Wightman, J.A. Jankowski, R.T. Kennedy, K.T. Kawagoe, T.J. Schroeder, D.J. Leszczyszyn, J.A. Near, E.J. Diliberto, Jr. and O.H. Viveros, Proc. Natl. Acad. Sci. USA, 1991, 88, 10754-10758.

21 C. Spégel, A. Heiskanen, L.H. Dæhli Skjolding and J. Emnéus, Electroanalysis, 2008, 20, 680-702.

11522 C. Krabbe, E. Courtois, P. Jensen, J.R. Jørgensen, J. Zimmer, A. Martinez-Serrano and M.J. Meyer, Neurochem., 2009, 110, 19081920.

23 A. Villa, I. Liste, E.T. Courtois, E.G. Seiz, M. Ramos, M. Meyer, B. Juliusson, P. Kusk and A. Martinez-Serrano, Exp. Cell Res., 2009, 315, 1860-1874.

24 R.F. Lane and A.T. Hubbard, Anal. Chem., 1976, 48, 1287-1293.

25 Z. Gao and A. Ivaska, Anal. Chim. Acta, 1993, 284, 393-404.

26 C. Spégel, A. Heiskanen, J. Acklid, A. Wolff, R. Taboryski, J. Emnéus and T. Ruzgas, Electroanalysis, 2007, 19, 263-271. 
27 E.M. Genies, G. Bidan and A.F. Diaz, Electroanal. Chem., 1983, 149, 101-113.

28 H. Ge, G. Qi, E-T. Kang and K.G. Neoh, Polymer, 1994, 35, 504508.

529 P.A. Christensen and A. Hamnett, Electrochim. Acta, 1991, 36, $1263-$ 1286.

30 K. Pihel, D. Walker and R.M. Wightman, Anal. Chem., 1996, 68, 2084-2089.

31 F. Beck, R. Michaelis, F. Schloten and B. Zinger, Electrochim. Acta, 1994, 39, 229-234.

32 T. Shimidzu, A. Ohtani, T. Iyoda and K. Honda, J. Electroanal. Chem., 1987, 224, 123-135.

33 K. Naoi, M. Lien and W.H. Smyrl, Electrochem. Soc., 1991, 138, 440-445.

1534 R. Yang, K. Naoi, D-F. Evans, W.H. Smyrl, W.A. Hendrickson, Langmuir, 1991, 7, 556-558.

35 L. Atanasoska, K. Naoi and W.H. Smyrl, Chem. Mater., 1992, 4, 988-994.

36 A. Dalmia, C.C. Liu and R.F. Savinell, J. Electroanal. Chem., 1997, 20 430, 205-214

37 R.H.S. Westerink and A.G. Ewing, Acta Physiol. (Oxf.), 2008, 192, 273-285.

38 L.A. Greene and A.S. Tischler, Proc. Natl. Acad. Sci. USA, 1976, 73, 2424-2428.

2539 F. Hefti, J. Hartikka and M. Schlumpf, Brain Res., 1985, 348, $283-$ 288.

40 P. Aebischer, M. Goddard, A.P. Signore and R.L. Timpson, Exp. Neurol., 1994, 126, 151-158.

41 P. Roach, T. Parker, N. Gadegaard and M.R. Alexander, Surf. Sci. 30 Rep., 2010, 65, 145-173.

42 J-H. Tao-Cheng, A. Dosemeci, J.P. Bressler, M.W. Brightman, D.L. Simpson, J. Neurosci. Res., 1995, 42, 323-334.

43 H-F. Cui, J-S. Ye, Y. Chen, S-C. Chong and F-S. Sheu, Anal. Chem., 2006, 78, 6347-6355.

3544 A. Heiskanen and J. Emnéus, "Monitoring of cellular dynamics with electrochemical detection techniques", in N. Eliaz (Ed.), Applications of Electrochemistry and Nanotechnology in Biology and Medicine I, Modern Aspects of Electrochemistry Vol. 52, Springer Science: New York, 2011, 1-102.

4045 C. Amatore, S. Arbault, M. Guille and F. Lemaître, Chem. Rev., $2008,108,2585-2621$.

46 K. Reimer, C. Köhler, T. Lisec, U. Schnakenberg, G. Fuhr, R. Hintsche and B. Wagner, Sens. Act. A, 1995, 46-47, 66-70.

47 L.M. Fischer, M. Tenje, A.R. Heiskanen, N. Masuda, J. Castillo, A.

45 Bentien, J. Emnéus, M.H. Jakobsen and A. Boisen, Microelectron. Eng., 2009, 86, 1282-1285.

48 L.A. Sombers, M.M. Maxson and A.G. Ewing, J. Neurochem., 2005, 93, 1122-1131.

49 M. Vergani, M. Carminati, G. Ferrari, E. Landini, C. Caviglia, A. Heiskanen, C. Comminges, K. Zór, D. Sabourin, M. Dufva, M. Dimaki, R. Raiteri, U. Wollenberger, J. Emnéus and M. Sampietro, IEEE Trans. Biomed. Circuits Syst., 2012, DOI 10.1109/TBCAS.2012.2187783.

50 C. Spégel, A. Heiskanen, S. Pedersen, J. Emnéus, T. Ruzgas and R. 55 Taboryski, Lab Chip, 2008, 8, 323-329. 


\section{Figures and Figure Captions}

5 Figure 1

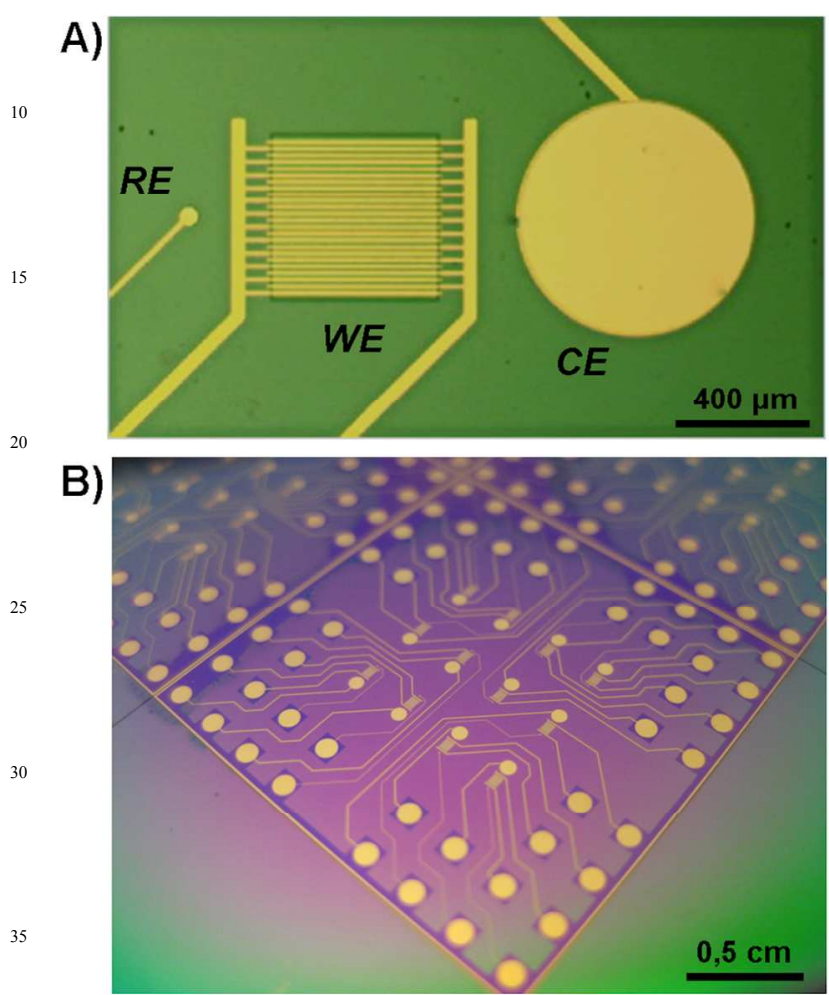

Fig. 1 A) An individual set of reference electrode (RE), working IDEs (WE) and counter electrode (CE); B) a 40 microfabricated chip containing 12 sets of electrodes with individual contact pads on the outskirts of the chip for electrical connections (for a complete view of the analytical system setup, see ESI $\uparrow$ ).

45

50

60

8 | Journal Name, [year], [vol], oo-oo
75

Figure 2
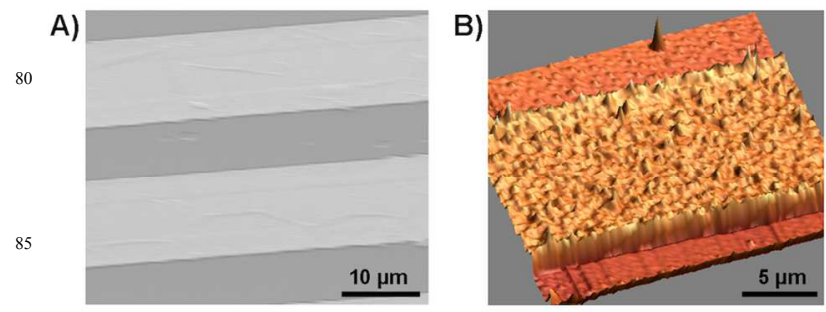

C)

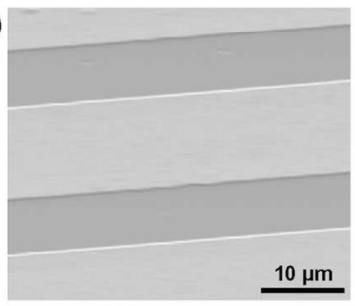

D)

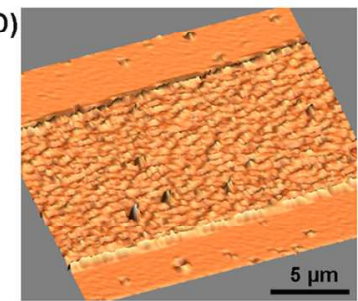

E)
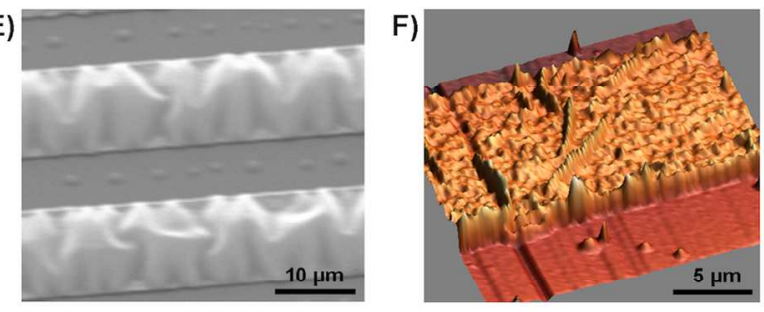

\section{G)}
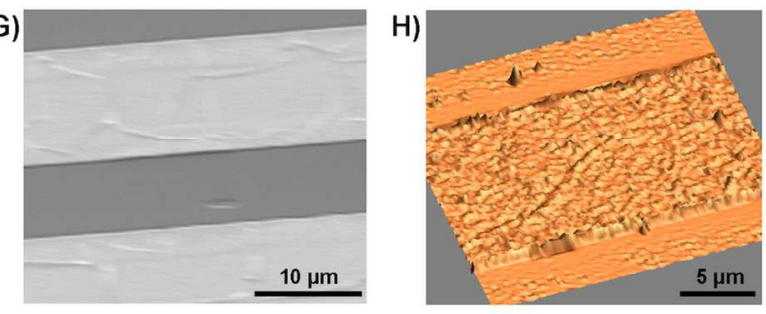

Fig. 2 SEM (A, C, E, G) and AFM (B, D, F, H) images of ${ }^{120}$ electropolymerized PPy films on IDEs, showing typical morphologies for A, B) small-counter ion doped films (in this case $\left.\mathrm{Cl}^{-}\right)$; $\left.\mathrm{C}, \mathrm{D}\right)$ overoxidized small-counter ion $\left(\mathrm{Cl}^{-}\right)$doped films; E, F) large-counter ion ( $\mathrm{PSS}^{-}$) doped films; $\mathrm{G}, \mathrm{H}$ ) ${ }_{125}$ overoxidized large-counter ion ( $\left.\mathrm{PSS}^{-}\right)$doped films. 
Figure 3

10

15

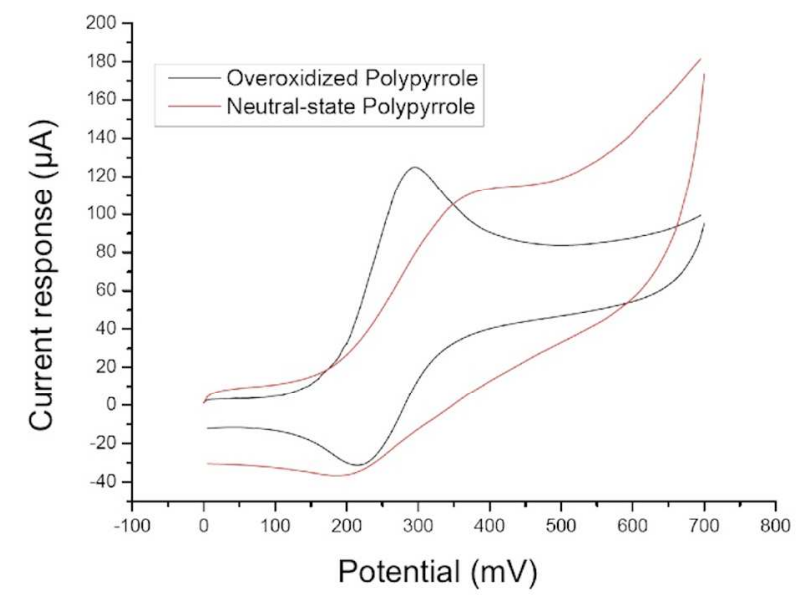

Fig. 3 Typical cyclic voltammograms of a polypyrrole

${ }^{30}$ modified gold IDE set in $100 \mu \mathrm{M}$ dopamine (in PBS) before and after overoxidation (no dopant counter-ions were added). The integrated RE and CE for the IDE set were used to obtain the three-electrode configuration. The potentials ${ }_{35}$ were adjusted vs. gold $\mathbf{R E}$, the potential sweep rate was $\mathbf{5 0}$ $\mathrm{mV} / \mathrm{s}$ (sweep direction from 0 to 700 to $0 \mathrm{mV}$ ).
40

Figure 4

80

85

90

95

105

110

100

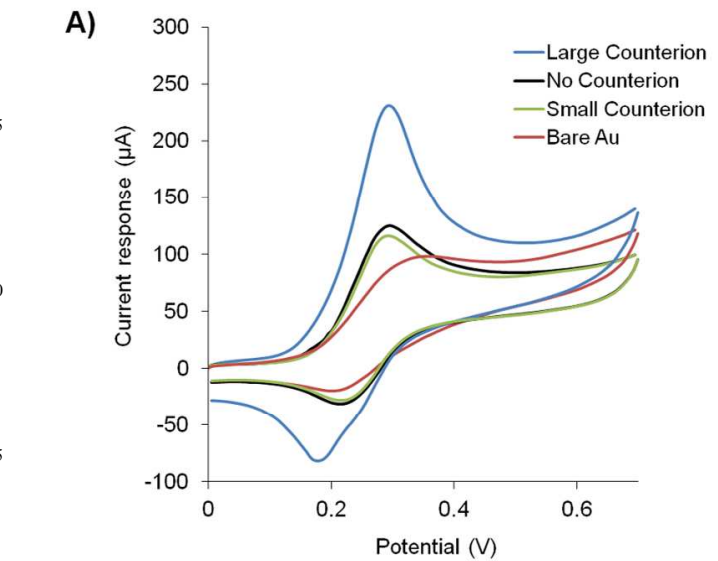

B)

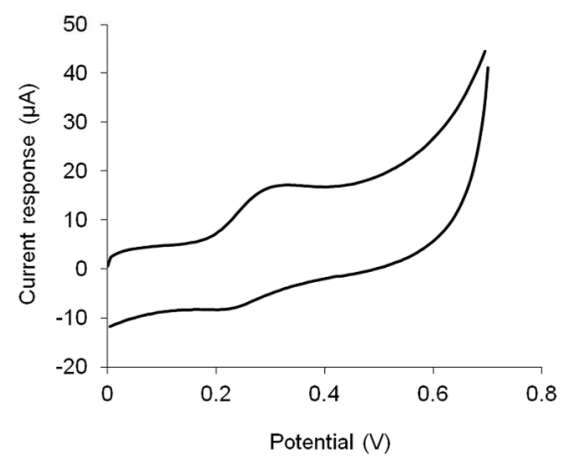

${ }_{115}$ Fig. 4 A) Comparison of typical cyclic voltammograms in $100 \mu \mathrm{M}$ dopamine (in PBS) from sets of IDEs modified with overoxidized PPy doped with different counter ions (Large Counterion refers to $\mathrm{PSS}^{-}$, and Small Counterion to $\mathrm{PO}_{4}{ }^{3-}$ ). The integrated RE and CE for each IDE set were used to 120 obtain the three-electrode configuration. The potentials were adjusted vs. gold RE and the potential sweep rate was $\mathbf{5 0}$ $\mathrm{mV} / \mathrm{s}$ (sweep direction from 0 to 0.7 to $0 \mathrm{~V}$ ). B) Voltammogram obtained from the overoxidized PPy/PSS modification as a response to $10 \mathrm{nM}$ dopamine. 
5

Figure 5

15

A)

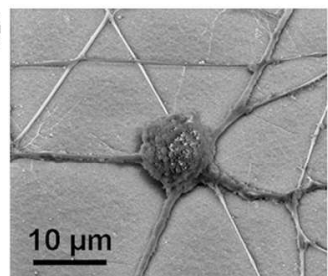

B)

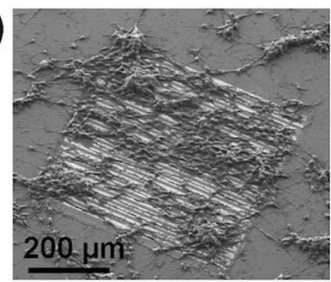

20

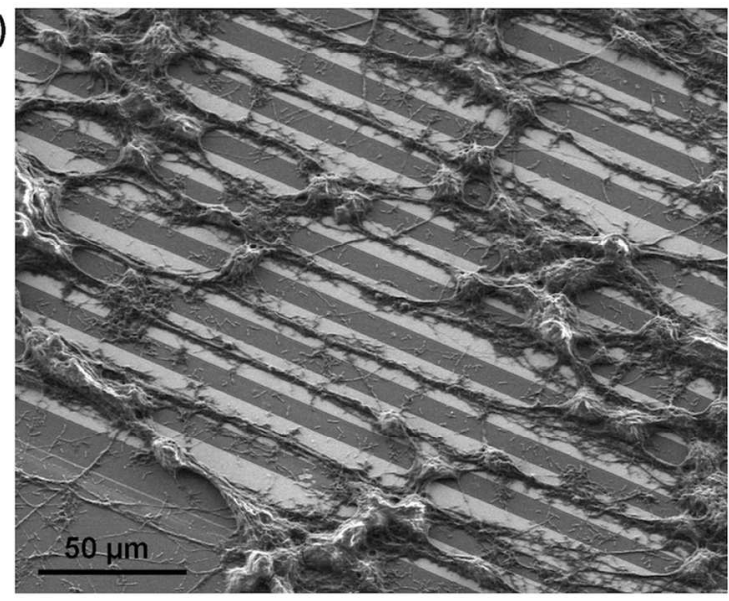

Fig. 5 SEM images of differentiated PC12 cells on PSS${ }^{40}$ doped overoxidized PPy-modified electrodes showing A) a single PC12 cell, B) a population of PC12 cells and C) a magnified view of the cell population in $B$.
Figure 6

80

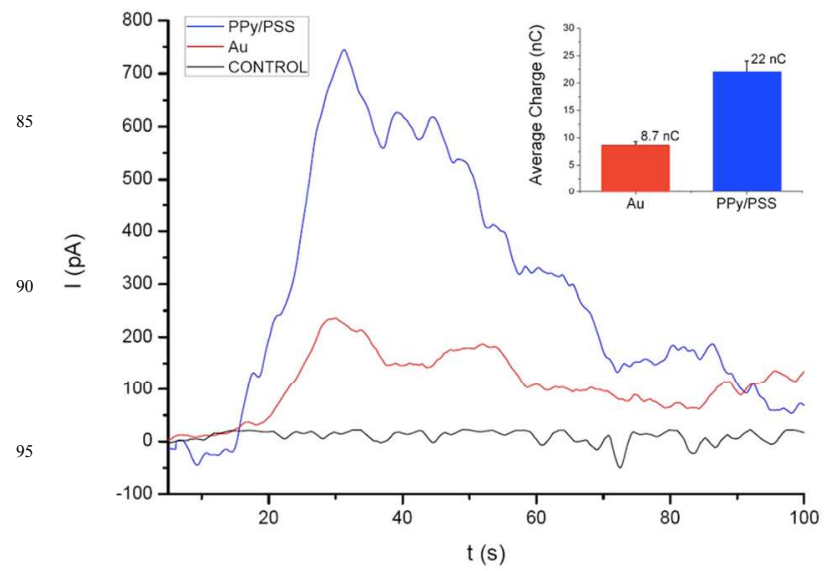

100 Fig. 6 Typical amperometric current-time traces, corresponding to dopamine release from differentiating PC12 cells, obtained during an exocytosis measurement on a bare gold IDE (red line, Au) and on an IDE modified with PSS $^{-}$-doped overoxidized PPy (blue line, PPy/PSS). The

${ }^{105}$ black line (CONTROL) corresponds to the signal recorded on an IDE without PC12 cells. Inset shows the average charge accumulated during measurements on non-modified (Au) and modified IDEs (PPy/PSS). The error bars ${ }_{110}$ represent s.e.m. $(\mathrm{n}=5)$. 


\title{
Table Of Contents (TOC) Entry
}

\section{Doped Overoxidized Polypyrrole Microelectrodes as Sensors}

\author{
for the Detection of Dopamine Released from Cell
}

\section{Populations}

\author{
Luigi Sasso, ${ }^{a}$ Arto Heiskanen, ${ }^{a}$ Francesco Diazzi, ${ }^{a}$ Maria Dimaki, ${ }^{a}$ Jaime Castillo- \\ León, ${ }^{a}$ Marco Vergani, ${ }^{b}$ Ettore Landini, ${ }^{c}$ Roberto Raiteri, ${ }^{c}$ Giorgio Ferrari, ${ }^{b}$ Marco \\ Carminati, ${ }^{b}$ Marco Sampietro, ${ }^{b}$ Winnie E. Svendsen ${ }^{* a}$ and Jenny Emnéus ${ }^{a}$
}

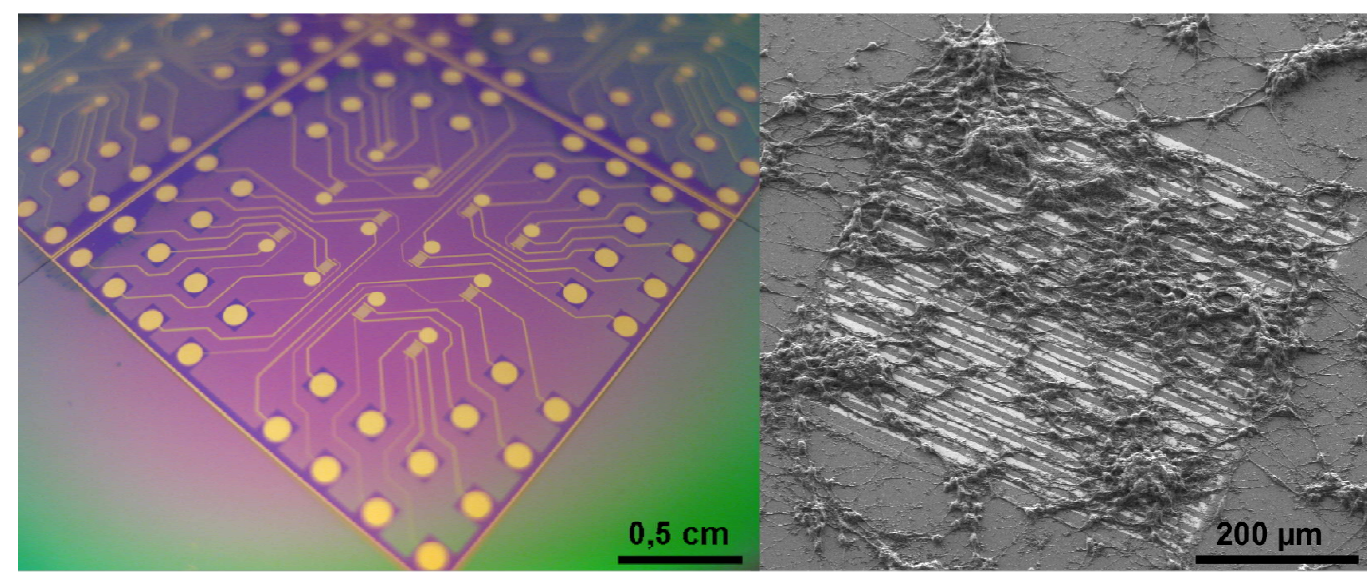

A microelectrode modification was developed using electropolymerized polypyrrole, testing several counter ion dopants for sensitivity increase towards dopamine, and used for the detection of dopamine from a population of cells. 